\title{
Manufacturing of polycaprolactone - Graphene fibers for nerve tissue engineering
}

\author{
Paola Ginestra \\ Dept. of Mechanical and Industrial Engineering, University of Brescia - V. Branze 38, 25123, Brescia, Italy
}

\section{A R T I C L E I N F O}

\section{Keywords:}

Electrospinning

Mechanical testing

Tissue engineering

\begin{abstract}
A B S T R A C T
Nanofibrous structures have morphological similarities to extracellular matrix and have been considered as candidate scaffolds in tissue engineering. Scaffolds made from electrospun fibers have potential in cell adhesion, proliferation and cell function. In this study, different percentages of graphene have been dispersed in a polycaprolactone-cyclopentanone solution to produce electrospun fibers. The microstructure and morphology of the fibers and the mechanical behavior of the electrospun systems were evaluated to analyze the influence of graphene content on the performances of the fibers. A significant dimensional difference between the fibers diameters of was obtained due to the graphene percentage. Accordingly, the mechanical properties of the fibrous systems are found to be influenced by the presence of the graphene. Rat stem cells were cultured on the fibrous scaffolds to evaluate the effect of the arrangement of the fibers on the morphology of the cells and differentiation into neurons. In particular, a higher population of dopaminergic neurons has been identified on the fibers with a higher percentage of graphene.
\end{abstract}

\section{Introduction}

Tissue engineering is currently focused on finding the combination of mechanical and biochemichal properties required for an engineered scaffold at multiple hierarchical scales (Johnson et al., 2009; Pham et al., 2006; Gastaldi et al., 2015). By designing biomaterials with specifically tuned pore structures, shapes, sizes and mechanical properties, researchers can stimulate and guide tissue repair and function restoration (Gunatillake and Adhikari, 2003; Ceretti et al., 2017a). Nerve tissue engineering is focusing on neural cells implants, considering the limited recovery capacity from damage that distinguishes the central nervous system. The biological substitutes aiming at restoring or improving nerve tissue function compromised by neurodegenerative disorders are increasing over the past few years (Prabhakaran et al., 2009; Ginestra et al., 2017a, 2019). Biocompatible scaffolds that mimic the biological environment of native Extra-Cellular Matrix (ECM) play an important role in supporting and regulating the differentiation of stem cells. Biocompatible scaffolds can be designed to promote the repair of the tissue in different manners starting from providing a physical support for the development and proliferation of the cells and finishing to the direct guidance of the cells growth (Ginestra et al., 2017b, 2017c). Artificial scaffolds consisting of nanofibers have large surface area and high porosity suitable to assure cells attachment and adhesion, showing that electrospinning is a potential strategy to produce biomimetic scaffolds (Ginestra et al., 2016a; Inverardi et al., 2018). The scaffolds produced by electrospinning are characterized by a 3D fibrous architecture and interconnected porosity that make them a suitable option for the neural tissue engineering (Wang et al., 2012). Functional electrospun scaffolds with high surface area to volume ratios can provide a suitable surface for the attachment and proliferation of Neural Stem Cells (NSC) improving their effectiveness in cell replacement therapies (Carlberg et al., 2009). A broad spectrum of nanofibrous scaffolds can be obtained by the use of different biodegradable polymers. The physical and chemical properties of the materials match the surrounding tissue providing the appropriate support for the cells for specific applications (Park et al., 2011). The versatility of the electrospinning technique allows the production of customized scaffolds that can be modified by properly choosing the material and changing the process parameters (Binan et al., 2014; Ghasemi-Mobarakeh et al., 2009). In particular, the insertion of specific nanoparticles in the electrospinning solution modifies the properties of the polymer nanofibers and the resulting composites are able to promote and drive the differentiation and maturation of the cells. Graphene nanosheets have been used for nerve tissue engineering considering their biocompatibility and their ability to modify cell growth. A nanocomposite in which the graphene is embedded in a polymer matrix has no toxic effect on the cellular population and potentially induces stem cells to preferentially differentiate into a specific lineage

E-mail address: paola.ginestra@unibs.it. 
(Weaver and Cui, 2015). The present paper is intended to illustrate porous scaffold production by electrospinning of a solution containing poly-( $\varepsilon$-caprolactone) (PCL) and graphene powder in different percentages. The influence of the graphene content on fibers morphology and mechanical performances under tensile tests is analyzed and the effect of the electrospun scaffolds composition on the differentiation of neural stem cells has been investigated.

\section{Materials and methods}

\subsection{Electrospinning tests}

Poly( $\varepsilon$-caprolactone) with an average molecular weight of $80,000 \mathrm{~g} /$ mol was dissolved in Cyclopentanone. A solution of $22 \mathrm{wt} \%$ concentration of PCL was stirred for $2 \mathrm{~h}$ using a digital hotplate at $40^{\circ} \mathrm{C}$. Graphene nanosheets were grinded to be used as graphene powder at different concentrations. In particular, the dispersions were prepared with graphene concentrations of 0,1 and $2 \mathrm{wt} \%$. All the listed materials were purchased from Sigma Aldrich ${ }^{\circledast}$. The effect of a good dispersion of the nanoparticles into the polymer solution is reported in (Ceretti et al., 2017b). Briefly, the graphene was dispersed in Cyclopentanone and sonicated for $1 \mathrm{~h}$. After the sonication, a good dispersion of the graphene was obtained by putting the suspension under stirring for $24 \mathrm{hrs}$. The polymer was added to the graphene solution and the PCL-graphene solution was kept under stirring for another $24 \mathrm{~h}$.

The electrospinning tests were performed using the following parameters set: flow rate of $1 \mathrm{ml} / \mathrm{h}$, needle tip - collector distance of $180 \mathrm{~mm}$, voltage of $20 \mathrm{kV}$, temperature of $35^{\circ} \mathrm{C}$ and relative humidity of $40 \%$. A G21 needle was used for all the tests. The tests were carried out for $5 \mathrm{~min}$ and repeated three times under the same conditions to check the system variability (Fig. 1).

The PCL-graphene fibers were evaluated under Keyance VK-X optical microscope and Zeiss LEO 1525 SEM. For each test, the diameter of the fibers was measured using the image processing software ImageJ (Rasband, 1997). High-resolution transmission electron microscopy (HRTEM; FEI, Titan 80-300 kV S/TEM) was used to visualize and evaluate the microstructure of the PCL-graphene samples.

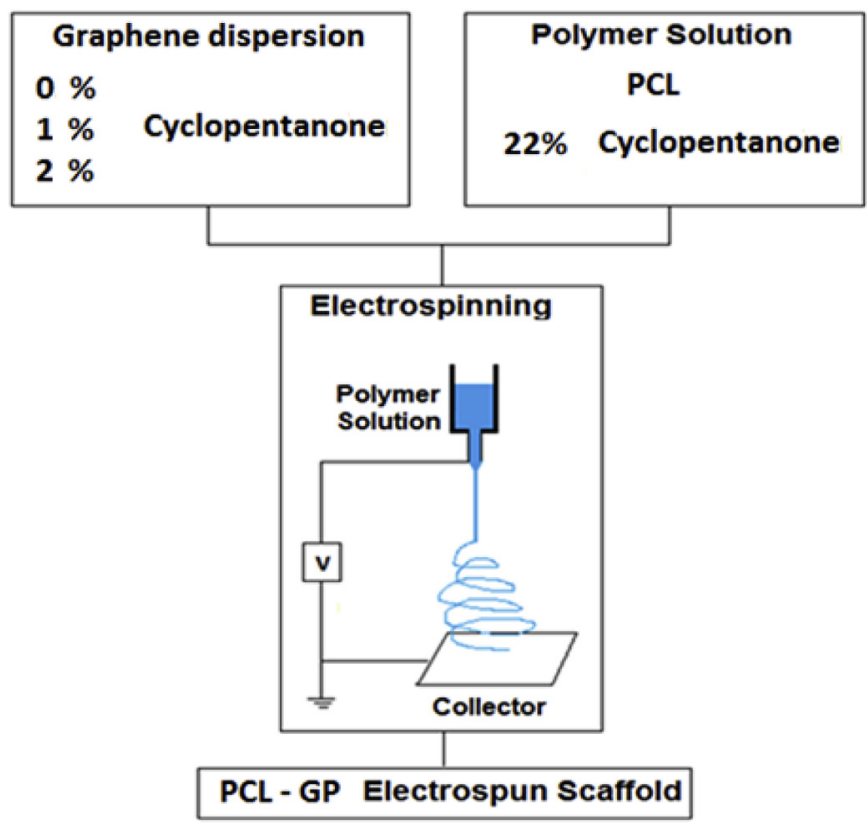

Fig. 1. Schematic representation of the experimental tests configuration.

\subsection{Scaffolds mechanical properties}

The mechanical properties of the scaffolds were analyzed as reported in (Ginestra et al., 2016b). Briefly, the mechanical behavior of the electrospun systems was evaluated under uniaxial tensile tests, carried out at $23^{\circ} \mathrm{C}$ by means of the dynamic mechanical analyzer DMA Q800 (TA Instruments). Rectangular strips were selected from the central portion of the electrospun area, with an overall length of $10 \mathrm{~mm}$ and a width of $5 \mathrm{~mm}$. The thickness of the samples increased when the graphene percentage within the polymer solution was increased ranging between 0.2 and $0.8 \mathrm{~mm}$. The tests were carried out with three repetitions for each specimen type. The samples were clamped carefully to avoid the failure of the fibrous mat at the gripping points, and then were subjected to a tensile ramp under displacement control at $0.2 \mathrm{~mm} /$ min until failure. The values of force $(F)$ and displacement $(\Delta \mathrm{L})$ recorded during the tests, were employed to evaluate stress $(\sigma)$ and strain $(\varepsilon)$ as engineering values, according to the following equations:

$\sigma=F / A_{0}$

$\varepsilon=\Delta \mathrm{L} / \mathrm{L}_{0}$

where $A_{0}$ and $L_{0}$ are the overall initial cross section and the initial distance between clamps, respectively.

The stress vs. strain relationship was determined for all the materials up to final break and the elastic modulus was calculated as the slope of the initial linear trend of the stress vs. strain curve.

\subsection{Biological tests}

Cell culture tests were performed using the scaffold characterized by different graphene percentages. The effects of the different parameters of the electrospun fibers were evaluated in terms of cell attachment and differentiation. Mouse E12 (StemCell Technologies ${ }^{\mathrm{TM}}$ ) stem cells were used for cell growth experiments. Cells were cultured using NeuroCult ${ }^{\mathrm{TM}}$ Proliferation medium supplemented with rh EGF (NeuroCult ${ }^{\mathrm{TM}}$ ). Cell proliferation medium contained Dulbecco's modified Eagle medium (Life Technologies $^{\mathrm{TM}}$ ), 1x B27 (Life Technologies ${ }^{\mathrm{TM}}$ ), 1x N2 (Life Technologies $^{\mathrm{TM}}$ ), $1 \mathrm{mM}$ sodium pyruvate (Life Technologies ${ }^{\mathrm{TM}}$ ), $2 \mathrm{mM} \mathrm{L-}$ glutamine (Life Technologies ${ }^{\mathrm{TM}}$ ), $1 \mathrm{mM}$ N-acetylcysteine (Sigma Aldrich $^{\circledR}$ ), and the following 3 growth factors: $20 \mathrm{ng} / \mathrm{ml}$ epidermal growth factor (EGF) (BD Biosciences), $10 \mathrm{ng} / \mathrm{ml}$ fibroblast growth factor (FGF) (BD Biosciences), and $2 \mathrm{mg} / \mathrm{ml}$ heparin (Sigma Aldrich ${ }^{\circledast}$ ). Differentiation medium was the same as the proliferation medium but the growth factors were removed. Cells were maintained at $37{ }^{\circ} \mathrm{C}$ in a saturated humidity atmosphere containing $95 \%$ air and $5 \% \mathrm{CO}_{2}$. Before cell seeding, all the scaffolds were washed twice with phosphate-buffered saline (PBS), and sterilized under UV light for $30 \mathrm{~min}$. The scaffolds were then coated with laminin and incubated overnight. Cultures were resuspended as single cells, and seeded at a cellular density of 40,000 cells $/ \mathrm{cm}^{2}$. Each scaffold was placed in a 24 well plate and the cells suspension was seeded onto the scaffolds' surface. The scaffolds were left in the incubator for $45 \mathrm{~min}$ before adding the proliferation medium. 3D printed cylindrical disks were placed on the samples to allow the fibrous mats to remain attached to the bottoms of the 24 well plates. The average thickness of the PCL-scaffold ranged between $0.2 \div 0.8 \mathrm{~mm}$. The proliferation medium was changed every two days. After two days of proliferation, the proliferation medium was changed to differentiation medium for three days.

The scaffolds were fixed in $4 \%$ paraformaldehyde for $10 \mathrm{~min}$. To determine what cell type NSCs differentiated into following stimulation, cells were stained with different antibodies and imaged using Nikon Eclipse Ti Fluorescent microscope and pictures were captured with NIS element AR3.10 software. Two types of antibodies were used for staining: anti- $\beta$ III-tubulin (Tuj-1) to stain all neurons, and antiTyrosine Hydroxylase to selectively stain dopaminergic neurons. For staining, all cells were treated with $0.3 \%$ Triton X-100 in PBS for 5 min 

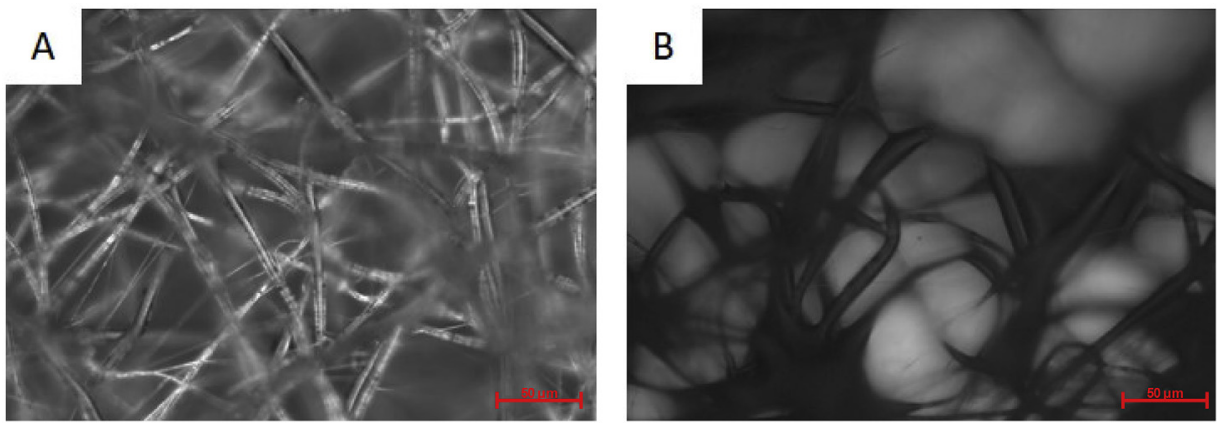

Fig. 2. Optical microscope images 500X of PCL electrospun fibers with (A) $1 \%$ and (B) $2 \%$ of Graphene.

prior to blocking for $1 \mathrm{~h}$ in $5 \%$ bovine serum albumin (BSA) in PBS followed by the overnight incubation at $4{ }^{\circ} \mathrm{C}$ with anti- $\beta$ III Tubulin and anti-Tyrosine Hydroxylase antibodies (Abcam) in the blocking buffer. After washing with D-PBS, cells were stained with Alexa Fluor ${ }^{\circledR} 488$ (Life Technologies $^{\mathrm{TM}}$ ) and Alexa Fluor ${ }^{\circledast} 594$ (Life Technologies $^{\mathrm{TM}}$ ) for $2 \mathrm{~h}$ in the dark. Scaffolds were mounted onto glass slides using Vectashield (Vector Labs) before imaging.

\section{Results and discussion}

The effects of the different percentage of graphene powder inserted in the polycaprolactone solution have been evaluated to firstly identify their influence on the electrospun fibers diameters and subsequently to establish how the presence of graphene was affecting the properties of the produced fibrous mats.

\subsection{Scaffold morphology}

The presence of the nanoparticles in the polymer fibers is clearly shown in the optical microscope images (Fig. 2). In particular, the graphene is distributed along the axis of the fibers and the particles are less visible when the percentage of powder is increased to $2 \%$.

HRTEM images are reported in Fig. 3. As shown, the micrographs reveal that the structure of the PCL-graphene fibers is highly disordered and randomly curled in presence of 1 and $2 \%$ of graphene. By comparison, the nanostructure of PCL-graphene at $2 \%$ of nanoparticles content appears to be in transition from an amorphous to a more graphitic material (Fig. 3d). Here, the carbon fringes are somewhat visible but still fragmented and randomly oriented.

The diameter of the fibers is strongly affected by the presence and the content of the graphene as confirmed by the SEM images (Fig. 4).

The measured diameters were analyzed to verify the possibility of using the normal distribution to describe the statistical trend of the resulting data. The probability plot test of Minitab ${ }^{\circledast} 18$ software evaluates how well the data follow a normal distribution and calculates the p-value accordingly. In particular, the test provides the percentage of fitting of the data with the normal distribution which ranges from $0 \%$ to $100 \%$ (the higher the percentage, the better the fitting). The variability of the data was evaluated in order to model the influence of the different graphene percentages on the diameter of the fibers. In particular, as shown in Fig. 5, the fibers diameter increases when the graphene content is increased as a result of the progressive less dispersion of the nanoparticles in the solvent. In fact, Fig. 5a shows that the graphene content influences both dimension and uniformity of the fibers. In particular, fibers are thicker and with a higher variability as the graphene percentage increases. The distribution of the fibers diameter (Fig. 5b) can be represented by a normal distribution which becomes less accurate as the graphene content increases (p-values from 0.210 for $2 \%$ of graphene to 0.020 for $0 \%$ of graphene).

The results of this analysis show that the dimension of the fibers seems to be responsible of the fiber deposition and arrangement on the collector resulting in a change of the fibers morphology.
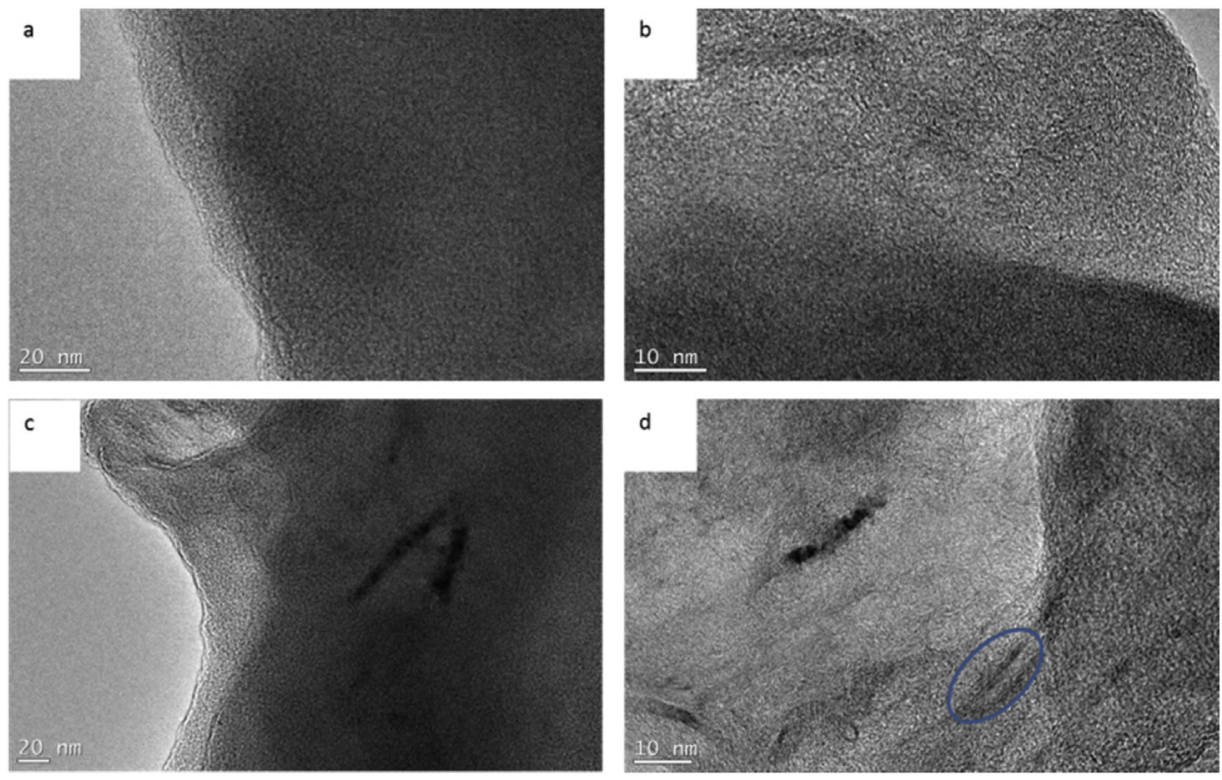

Fig. 3. HRTEM micrographs of the nanofibers with different graphene percentages: a,b) $1 \%$, c,d) $2 \%$. 

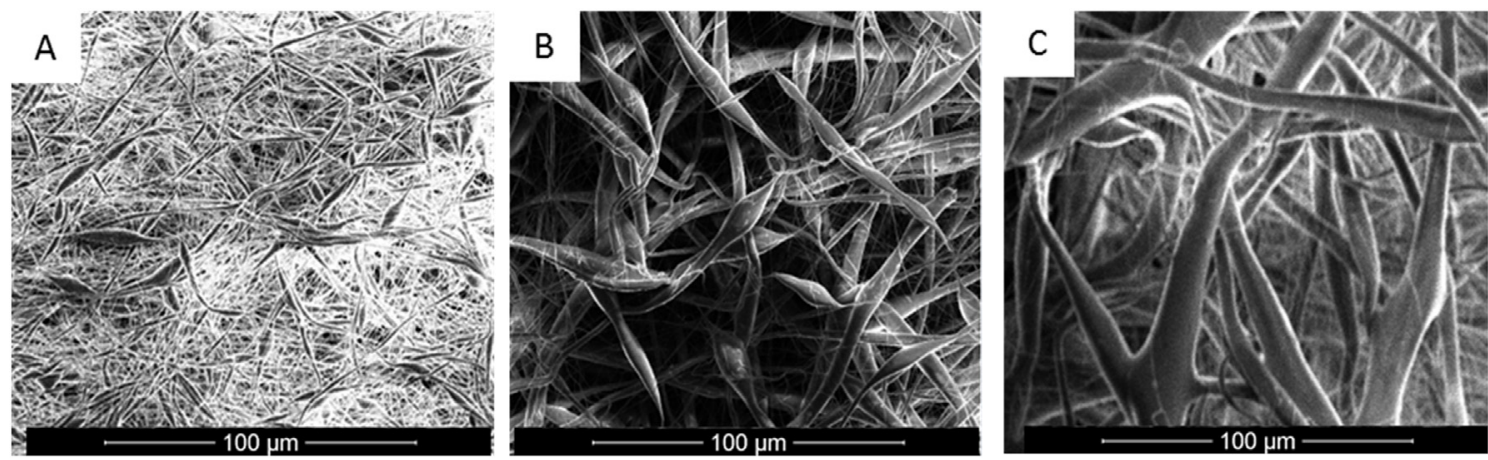

Fig. 4. SEM images $500 \mathrm{X}$ of the nanofibrous scaffolds produced with different graphene percentages: A) $0 \%$, B) $1 \%$ and C) $2 \%$.

\subsection{Mechanical properties}

The results of the mechanical tests are reported in Fig. 6, as the most representative nominal stress vs. nominal strain curve for each material group.

The mechanical response of the material seems to be strongly affected by the presence of the graphene. The failure area of the samples was observed corresponding to the central region of each strip along the cross-sectional area. The electrospun fibers are showing a peculiar behavior consisting of non-regular trends at high levels of strain in which several peaks are found as a consequence of a progressive and simultaneous restructuring and damaging of the electrospun. In particular, the graphene content seems to induce the progressive necking followed by the failure of small fibrous regions with a subsequent loss of the cross-sectional area subjected to the tensile load. According to the observed behavior, a regular increase of the stress is observed till the maximum values of stresses are reached between the $25-45 \%$ of strain, probably due to the necking of the cross sections and local failure events. In particular, the maximum stress (maximum point of stress) calculated in absence of graphene is equal to $1.46 \pm 0.1 \mathrm{MPa}$ while when the percentage of nanoparticles is $1 \%$ the maximum stress increases to $3.48 \pm 0.2 \mathrm{MPa}$ and in presence of $2 \%$ of graphene is equal to $2.25 \pm 0.2 \mathrm{MPa}$. Accordingly, the yield stress (evaluated as off-set yield strength corresponding to the $25 \%$ of strain) is significantly different depending on the percentage of nanoparticles: it is increased to $3.14 \pm 0.4 \mathrm{MPa}$ when a $1 \%$ of graphene is used, while it is equal to $1.96 \pm 0.2 \mathrm{MPa}$ at a graphene percentage of $2 \%$ and $1.32 \pm 0.1 \mathrm{MPa}$ in absence of nanoparticles. A progressive decrease of the stress is visible from Fig. 6, due to additional failure events which lead to high elongations at break for each material group. The presence of graphene is increasing the elastic modulus value to $21 \pm 3 \mathrm{MPa}$ and $22.5 \pm 5 \mathrm{MPa}$ respectively for $1 \%$ of graphene and $2 \%$ of graphene, while in absence of graphene the elastic modulus value is equal to $5.6 \pm 2 \mathrm{MPa}$. The elastic modulus of the composite material is probably influenced by the nanoparticles dispersion into the electrospinning solution that is causing the increase of the diameter of the fibers.

It can be concluded that, as shown from the graphs in Fig. 6, the mechanical properties can be modified by the insertion of different percentages of graphene. The comparison of the results shows that the insertion of the graphene leads to an increasing strength of the material that appears more resistant when the graphene percentage is $1 \%$. On the other hand, the mechanical properties seem to decrease in terms of elastic behavior and ultimate tensile strength with a higher graphene content. In particular, the influence of the graphene on the polymer fibers behavior starts to cause a deterioration of the mechanical response at a percentage of $2 \%$, showing a possible saturation of the solution corresponding to a poor dispersion of the nanoparticles within the solvent, leading to bigger deposited fibers in a reduced deposition area. As reported (Kim et al., 2010a), the mechanisms of interaction between the nanoparticles and the polymer that influences the mechanical response of such matrices has to be fully understood. Although the changes on the material stiffness caused by graphene have already been found to be more evident for elastomers (Kim et al., 2010b), in this case the influence of the matrix reinforcement made by the electrospinning of the graphene-polymer solutions is probably due to the induced spatial distribution of the anisotropic inclusions. Despite this study concerns with the elastic deformations at relatively low levels of loading due to the typical necking of the composite material (Young et al., 2018), the possibility of tailoring the mechanical properties of the electrospuns, is a promising step towards a full understanding of the
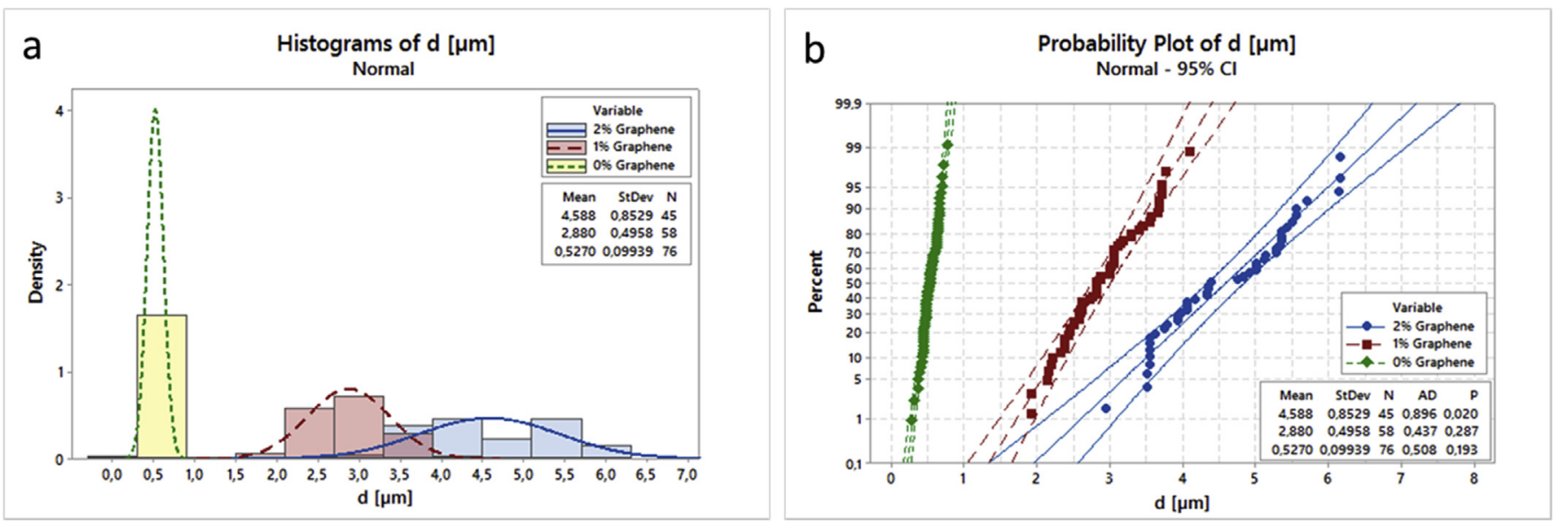

Fig. 5. a) Histograms of the frequency of the diameter of the fibers at different graphene percentages. b) Probability plots of the diameter of the fibers the collected data can be represented by a normal distribution. 


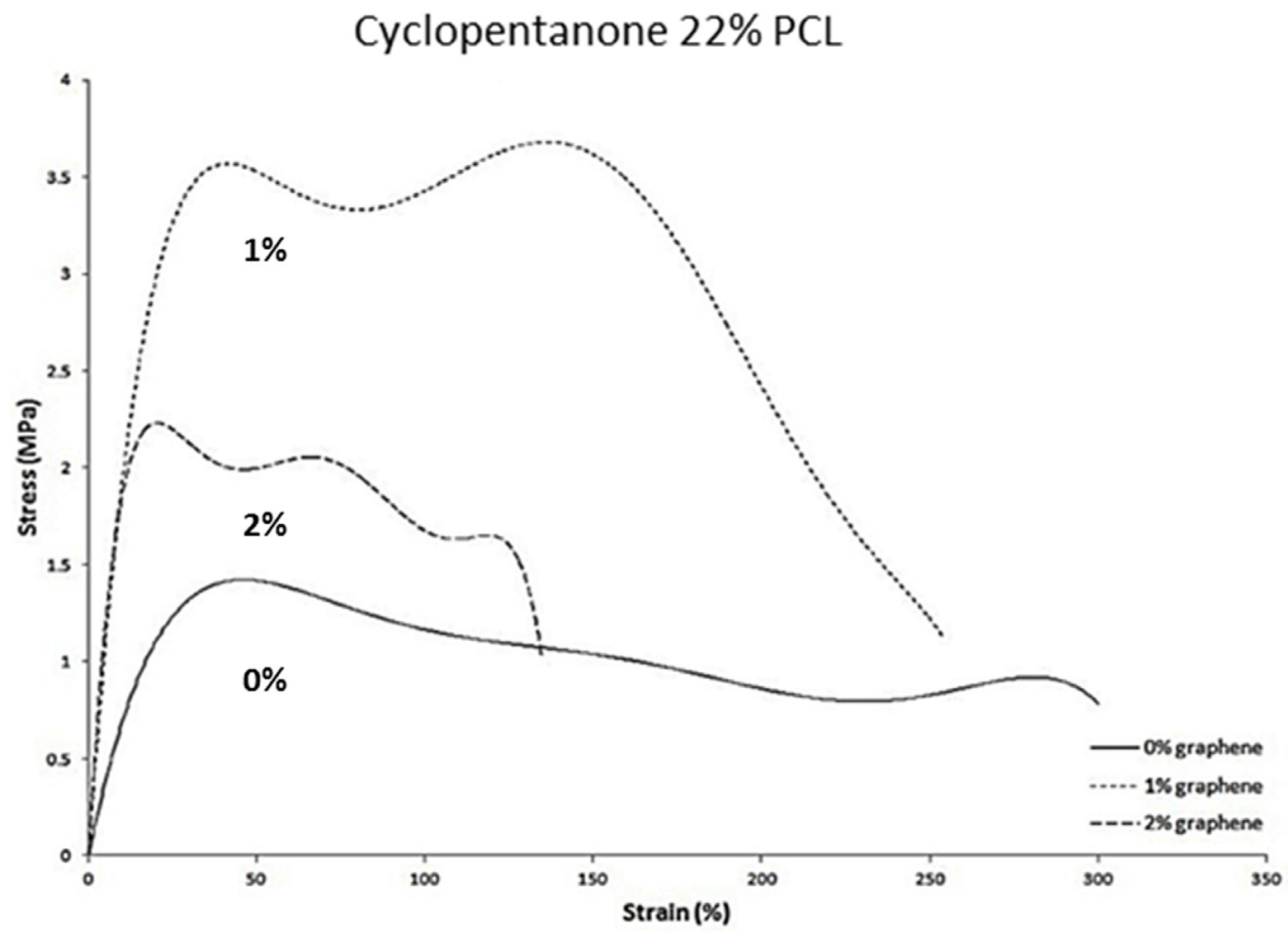

Fig. 6. Tensile nominal stress $v s$. nominal strain curves for the electrospun PCL with different contents of graphene. The reported curves refer to the representative sample for each material group.
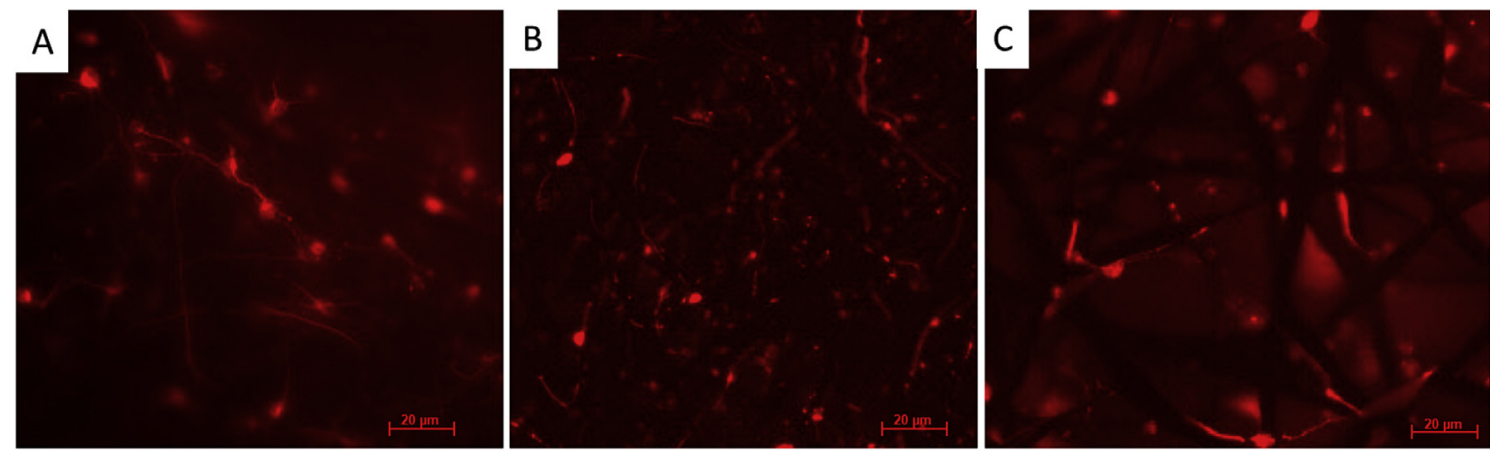

Fig. 7. Fluorescent microscopy 20X images of differentiated neurons recognized on the scaffolds produced with different graphene percentages: A) $0 \%$, B) $1 \%$ and C) $2 \%$.
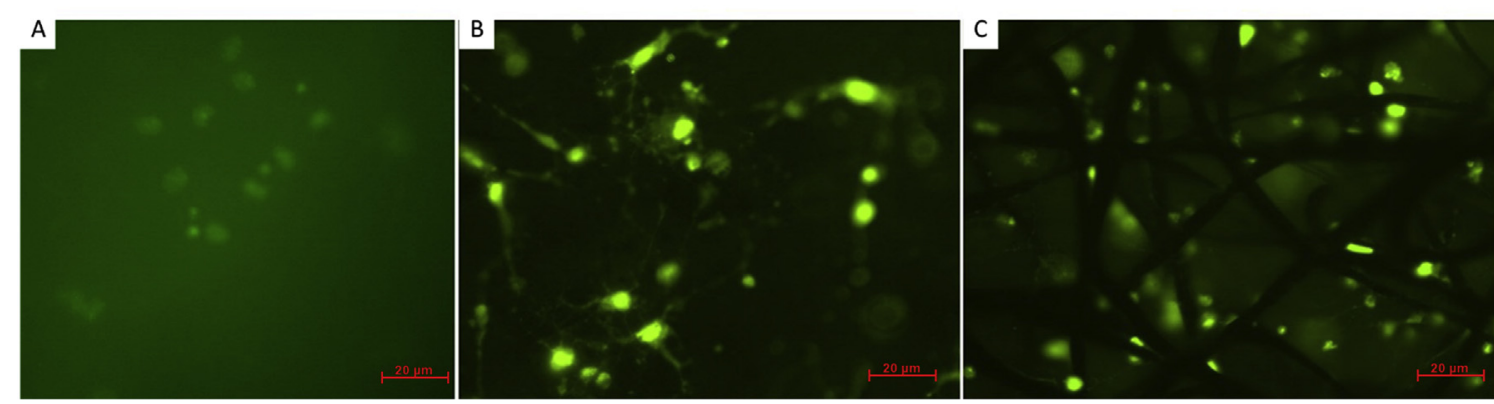

Fig. 8. Fluorescent microscopy $20 \mathrm{X}$ images of dopaminergic neurons recognized on the scaffold produced with different graphene percentages: A) $0 \%$, B) $1 \%$ and C) $2 \%$.

mechanical performance of reinforced polymer fibers on the base of the dispersion process.

\subsection{Cell differentiation}

The differentiation of the cells was evaluated after 5 total days of culture. The samples were observed with an inverted fluorescent 

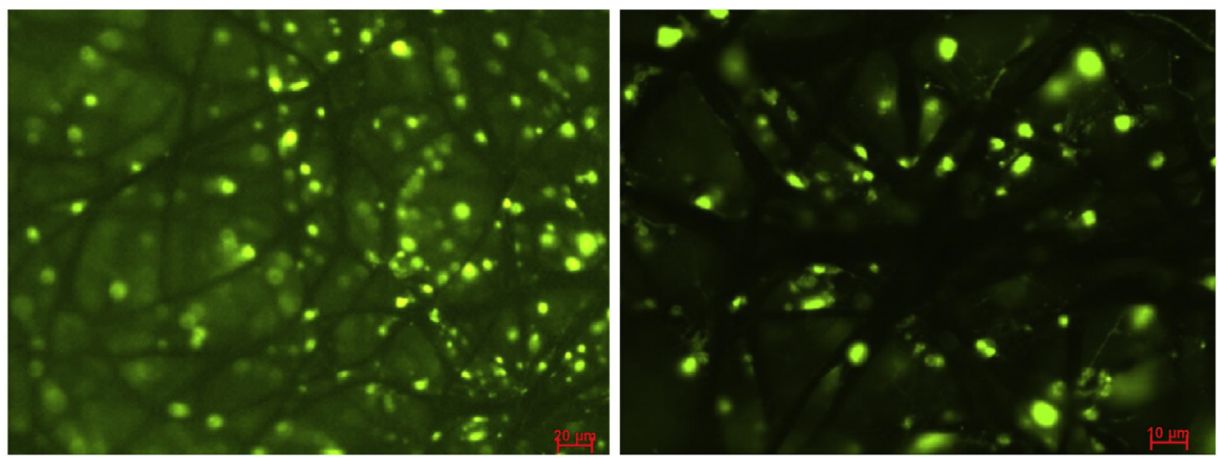

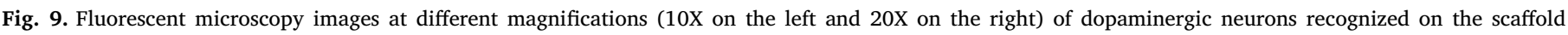
produced with $2 \%$ of graphene.

microscope at $10 \mathrm{x}$ and $20 \times$ magnifications and 5 images for each sample were taken. The orientation of the protein Class III $\beta$-tubulin was evaluated to analyze the interaction of the cells with the electrospun fibers and separate neurons from the glial cells.

Fig. 7 shows the results of the differentiation of the cells on the scaffolds with 0,1 and $2 \%$ of graphene.

As reported in Fig. 7, the protein Class III $\beta$-tubulin orientation is showing the differentiation of the cells on the scaffolds produced without graphene (A) and with $1 \%$ (B) and $2 \%$ (C) of graphene. As a matter of fact, it seems that the stem cells are differentiated in neurons on all the analyzed scaffolds with no differences connected to the presence of graphene. On the other hand, the cells seem to lie directly on the fibers of the PCL-graphene scaffolds while in absence of the nanoparticles the cells are colonizing the surface of the fibrous network.

The presence of dopaminergic neurons on the different substrates was then verified. The presence of Tyrosine Hydroxylase was evaluated to recognize the dopaminergic neurons resulting from the differentiation. The stained cells are reported in Fig. 8, where the difference between the substrates is evident.

The differentiation of the stem cells in dopaminergic neurons seems to be strongly affected by the presence of graphene. In particular, the dopaminergic neurons are not uniformly present on the PCL surface and Fig. 8A reports the only group of cells that was possible to identify in absence of graphene. The presence of the graphene seems to enhance the differentiation of the neural stem cells in dopaminergic neurons (Fig. 8B and C). Moreover, the dopaminergic neurons are colonizing all the fibers in a three-dimensional way within the PCL scaffolds with $2 \%$ of graphene as Fig. 9 points out at different magnifications:

The results of the staining suggest that the electrospun PCL-graphene nanofibers supported the differentiation of neural cells, creating the possibility of cell-scaffold transplantation of a construct with differentiated neuronal cells to the site of nerve injury. In this work, the scaffolds were made by randomly oriented fibers, however, the neurons showed a neutrite outgrowth typical of the cells seeded onto aligned electrospun fibers (Lim et al., 2010). The diameter of the fibers is promoting the differentiation of the neural stem cells in the neuronal lineage as previously shown (Christopherson et al., 2009) and the differentiation of the neurons showed an arrangement of the protrusions of the cells accordingly with the fibers arrangement and diameter. In this case, the higher diameter of the fibers in presence of graphene can promote the cellular colonization of the inner structure of the scaffolds instead of the surface as typical of the electrospun substrates used for nerve tissue enigneering (Mahairaki et al., 2011). Moreover, the scaffolds with graphene promote the differentiation of the cells into dopaminergic neurons while the polymer scaffolds are not providing a suitable substrate for their development. Such nanoparticles have already been demonstrated to change the interaction of the cells with the scaffold due to the increased conductivity and tailorable mechanical properties (Sung et al., 2011; Li et al., 2013). In this case, the neurons are differentiating in dopaminergic cells without the insertion of specific brain-derived grow factors or the usage of inducing activity methods (Dehua et al., 2014; Nishat et al., 2018). Moreover, in this work has been highlighted that the amount of cells that are expressing the production of dopamine in the differentiated population is higher in relation with a higher graphene percentage within the fibrous structure.

\section{Conclusions}

The porous structures produced by electrospinning with high ratio between surface area and volume, are ideally useful in applications such as scaffold implants. Here, a new optimization process to produce functional electrospun scaffolds depending on different polymer-graphene combinations, to embed neural cells in suitable matrices that support cell adhesion and differentiation was proposed. Graphene based nanomaterials have been highlighted as promising substrates for neuronal differentiation of stem cells, neural regeneration and electrodes for neural recordings (Bei et al., 2019). Graphene enriched scaffolds demonstrated to increase neural differentiation by an enhanced growth and extension of neurons and the delivery of electrical stimulation (Niu et al., 2018). The developed research shows that the presence of the graphene affects the properties of the produced fibrous mats. In particular, the graphene percentage is strongly affecting the morphology and distribution of the fibers. Furthermore, the graphene percentage is a core variable for controlling the mechanical performances of the electrospun fibers. Although the effects of only graphene percentage have been studied, many of the basic mechanisms controlling materials bonding and structure formation have not been completely understood. The resulting scaffolds support stem cells adhesion and proliferation, thus being highly biocompatible. In particular, the structure of the deposited fibers has been demonstrated to influence the cellular recognition of the material and the cellular spreading within the fibrous structure. The insertion of the graphene particles seems to be strongly promoting the differentiation of the stem cells into dopaminergic neurons. On the other hand, the fluorescence images of the same cells on the pure PCL fibers show that dopaminergic neurons are difficult to identify and barely attached onto the scaffold surface. Overall, the data in this study suggested that the PCL-graphene fibrous substrates might be potentially suitable for clinical applications, such as nerve tissue engineering, nerve endoprostheses and nerve injury treatments. Moreover, the electrospun fibers developed in this study can be used as a valid substrate for the in vitro manipulation and treatment of the cells.

\section{References}

Bei, H.P., Yang, Y., Zhang, Q., et al., 2019. Graphene-based nanocomposites for neural tissue engineering. Molecules 24, 658-673.

Binan, L., Tendey, C., De Crescenzo, G., et al., 2014. Differentiation of neuronal stem cells into motor neurons using electrospun poly-L-lactic acid/gelatin scaffold. Biomat 35, 
664-674.

Carlberg, B., Axell, M.Z., Nannmark, U., et al., 2009. Electrospun polyurethane scaffolds for proliferation and neuronal differentiation of human embryonic stem cells. Biomed. Mater. 4, 045004.

Ceretti, E., Ginestra, P., Neto, P.I., et al., 2017a. Multi-layered scaffolds production via Fused Deposition Modeling (FDM) using an open source 3D printer: process parameters optimization for dimensional accuracy and design reproducibility. Proc CIRP 65, 13-18.

Ceretti, E., Ginestra, P.S., Ghazinejad, M., et al., 2017b. Electrospinning and characterization of polymer-graphene powder scaffolds. CIRP Ann. 66 (1), 233-236.

Christopherson, G.T., Song, H., Mao, H.Q., 2009. The influence of fiber diameter of electrospun substrates on neuronal stem cell differentiation and proliferation. Biomat 30, 556-564.

Dehua, Y., Ting, L., Minghan, X., et al., 2014. Graphene oxide promotes the differentiation of mouse embryonic stem cells to dopamine neurons. Nanomedicine 9, 16.

Gastaldi, D., Parisi, G., Lucchini, R., et al., 2015. A predictive model for the elastic properties of a collagen-hydroxyapatite porous scaffold for multi-layer osteochondral substitutes. Int. J. Appl. Mech. 7 (4). https://doi.org/10.1142/S1758825115500635.

Ghasemi-Mobarakeh, L., Morshed, M., Karbalaie, K., et al., 2009. The thickness of electrospun poly ( $\varepsilon$-caprolactone) nanofibrous scaffolds influences cell proliferation. Int. J. Artif. Organs 32, 150-158.

Ginestra, P., Ceretti, E., Fiorentino, A., 2016a. Electrospinning of poly-caprolactone for scaffold manufacturing: experimental investigation on the process parameters influence. Proc CIRP 49, 8-13.

Ginestra, P.S., Ghazinejad, M., Madou, M., et al., 2016b. Fabrication and characterization of polycaprolactone-graphene powder electrospun nanofibers. In: Proc. SPIE 9932, Carbon Nanotubes, Graphene, and Emerging 2D Materials for Electronic and Photonic Devices IX, 99320A.

Ginestra, P.S., Ferraro, R.M., Lanzi, G., et al., 2017a. Production of micro-patterned substrates to direct human iPSCs-derived neural stem cells orientation and interaction. Proc CIRP 65, 225-230.

Ginestra, P., Fiorentino, A., Ceretti, E., 2017b. Micro-structuring of titanium collectors by laser ablation technique: a novel approach to produce micro-patterned scaffolds for tissue engineering applications. Proc CIRP 65, 19-24.

Ginestra, P., Pandini, S., Fiorentino, A., et al., 2017c. Microstructured scaffold for cellular guided orientation: PCL electrospinning on laser ablated titanium collector. CIRP J. Manuf .Sci. Technol. 19, 147-157.

Ginestra, P.S., Madou, M., Ceretti, E., 2019. Production of carbonized micro-patterns by photolithography and pyrolysis. Precis. Eng. 55, 137-143.

Gunatillake, P.A., Adhikari, R., 2003. Biodegradable synthetic polymers for tissue engineering. Eur. Cells Mater. 5, 1-16.

Inverardi, N., Ginestra, P.S., Ferraro, R.M., et al., 2018. Shape memory electrospun nonwovens based on crosslinked poly(e-caprolactone) for multifunctional biological applications. In: AIP Conference Proceedings. 1981, 02006.

Johnson, J., Niehaus, A., Nichols, S., Lee, D., 2009. Electrospinning PCL in vitro: a microstructural basis for mechanical property change. J. Biomater. Sci. 20, 467-481.

Kim, H., Abdala, A.A., Macosko, C.W., 2010a. Graphene/polymer nanocomposites Macromolecules 43, 6515-6530.

Kim, H., Miura, Y., Macosko, C.W., 2010b. Graphene/polyurethane nanocomposites for improved gas barrier and electrical conductivity. ACS Publications 22, 3441-3450.

Li, N., Zhang, Q., Gao, S., et al., 2013. Three-dimensional graphene foam as a biocompatible and conductive scaffold for neural stem cells. Sci. Rep. 3, 1604.

Lim, S.H., Liu, X.Y., Song, H., et al., 2010. The effect of nanofiber-guided cell alignment on the preferential differentiation of neural stem cells. Biomat 31, 9031-9039.

Mahairaki, V., Lim, S.H., Christopherson, G.T., et al., 2011. Nanofiber matrices promote the neuronal differentiation of human ebryonic stem cell-derived neural precursors in vitro. Tissue Eng. A 17, 855-863.

Nishat, T., Vikram, T., Munmun, C., 2018. The efficacy of graphene foams for culturing mesenchymal stem cells and their differentiation into dopaminergic neurons. Stem Cell. Int. 2018, 12 Article ID 3410168.

Niu, Y., Chen, X., Yao, D., Peng, G., Liu, H., Fan, Y., 2018. Enhancing neural differentiation of induced pluripotent stem cells by conductive graphene/silk fibroin films. J. Biomed. Mater. Res. Part A 2018 106A, 2973-2983.

Park, S.Y., Park, J., Sim, S.H., et al., 2011. Enhanced differentiation of human neural stem cells into neurons on graphene. Adv. Mater. 23, 263-267.

Pham, Q.P., Sharma, U., Mikos, A.G., 2006. Electrospinning of polymeric nanofibers for tissue engineering applications: a review. Tissue Eng. 12, 1197.

Prabhakaran, M.P., Venugopal, J.R., Ramakrishna, S., 2009. Mesenchymal stem cel differentiation to neuronal cells on electrospun nanofibrous substrates for nerve tissue engineering. Biomat 30, 4996-5003.

Rasband WS. ImageJ. 1997-2015. U. S. National Institutes of Health, Bethesda, Maryland, USA.

Sung, Y.P., Jaesung, P., Sunh, H.S., et al., 2011. Enhanced differentiation of human neural stem cells into neurons on graphene. Adv. Mater. 23, H263-H267.

Wang, T.Y., Forsythe, J.S., Parish, C.L., et al., 2012. Biofunctionalisation of polymeric scaffolds for neural tissue engineering. J. Biomater. Appl. 27 (4), 369-390.

Weaver, C.L., Cui, X.T., 2015. Directed neural stem cell differentiation with a functionalized graphene oxide nanocomposite. Adv. Healthc. Mater. 4, 1408-1416.

Young, R.J., Liu, M., Kinloch, I.A., et al., 2018. The mechanism of reinforcement of polymers by graphene nanoplatelets. Compos. Sci. Technol. 154, 110-116. 